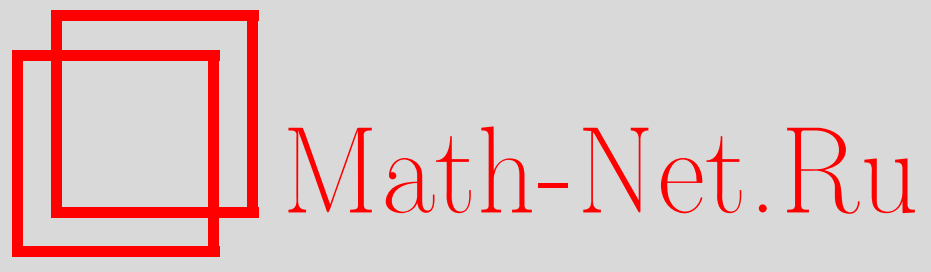

Я. Н. Шитов, О булевых матрицах полного факторизационного ранга, Матем. сб., 2013, том 204, номер 11, 151-160

DOI: https://doi.org/10.4213/sm8154

Использование Общероссийского математического портала Math-Net.Ru подразумевает, что вы прочитали и согласны с пользовательским соглашением http://www . mathnet.ru/rus/agreement

Параметры загрузки:

IP: 54.205 .225 .156

26 апреля 2023 г., 17:09:53

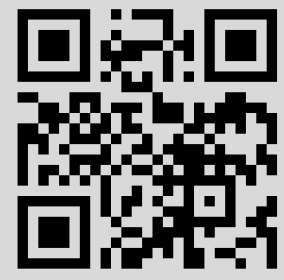




\title{
Я.Н. Шитов
}

\section{О булевых матрицах полного факторизационного ранга}

\begin{abstract}
Показано, что каждая $(0,1)$-матрица размера $n \times m$, имеющая булев ранг $n$, содержит столбец, в котором по крайней мере $\sqrt{n} / 2-1$ элементов равны нулю. Доказано, что приведенная оценка является асимптотически оптимальной. В качестве приложения полученных результатов показано, что булевы матрицы полного ранга не могут иметь сколь угодно большого размера при условии ограниченности их тропических или минорных рангов.

Библиография: 16 названий.
\end{abstract}

Ключевые слова: $(0,1)$-матрицы, булев ранг, число изоляции.

DOI: $10.4213 / \mathrm{sm} 8154$

\section{§ 1. Введение}

Булевым полукольцом называется множество $\mathbb{B}=\{0,1\}$ с операциями сложения $\oplus$ и умножения $\otimes$ соответственно, определяемыми следующим образом:

$$
a \oplus b=\max \{a, b\}, \quad a \otimes b=a b .
$$

В работе рассматриваются векторы и матрицы с элементами из полукольца $\mathbb{B}$. Через $A(i \mid j)$ будем обозначать элемент, стоящий в строке матрицы $A$, имеющей индекс $i$, и в столбце, имеющем индекс $j$. Арифметические операции над булевыми векторами и матрицами определяются так, как классические операции сложения и умножения, только операции + и · заменяются операциями $\oplus$ и $\otimes$ соответственно. Например, произведение $B \otimes C$ матриц $B \in\{0,1\}^{n \times k}$ и $C \in\{0,1\}^{k \times m}$ определяется как матрица $A$ размера $n \times m$ с элементами

$$
A(i \mid j)=\bigoplus_{t=1}^{k}(B(i \mid t) \otimes C(t \mid j)) .
$$

Важную роль для изучения матриц над полукольцом играет понятие ранга матрицы. Помимо классической ранговой функции оказывается полезным рассмотрение некоторых других ранговых концепций. В частности, одной из важнейших в полукольцевой линейной алгебре является функция факторизационного ранга (см. [1]), эта функция обобщает на случай матриц с элементами из произвольного полукольца классическое понятие ранга (см. [2]). Факторизационный ранг матрицы над полукольцом неотрицательных вещественных чисел известен как неотрищательный ранг матрицы и имеет множество приложений в квантовой механике, статистике, демографии и многих 
других областях (см. [3]). Полезно также рассматривать факторизационный ранг в случае матриц над тропическим полукольцом ввиду важных приложений в геометрии и комбинаторной оптимизации (см. [4]). Необходимость изучения понятия факторизационного ранга для булевых матриц обусловлена его приложениями в комбинаторике, поскольку эта ранговая функция играет важную роль в теории графов. А именно, рассматриваемая ранговая концепция дает линейно-алгебраическую характеризацию понятия двудольной размерности (см. [5]) графа: действительно, факторизационный ранг матрицы смежностей двудольного графа $G$ равен минимальной мощности покрытия графа $G$ полными двудольными подграфами этого графа (см. [6], [7]). Двудольная размерность графа представляет собой важный комбинаторный инвариант и находит широкое применение в приложениях, в частности для решения проблем компьютерной безопасности (см. [8]) и задач вычислительной математики (см. [9]). Теперь определим понятие факторизационного ранга матрицы, который в случае матриц над бинарным булевым полукольцом обычно называется их булевым рангом.

ОПРЕДЕЛЕНИЕ 1. Булевъм рангом матрицы $A \in\{0,1\}^{n \times m}$ называется минимальное целое число $k$, для которого найдутся матрицы $B \in\{0,1\}^{n \times k}$ и $C \in\{0,1\}^{k \times m}$, удовлетворяющие условию $A=B \otimes C$. Булев ранг матрицы, состоящей только из нулевых элементов, по определению полагается равным нулю.

Положив $B=A$ и в качестве $C$ взяв единичную матрицу порядка $m$, мы докажем, что булев ранг матрицы $A \in\{0,1\}^{n \times m}$ не превосходит $m$; можно показать аналогично, что этот ранг не превосходит $n$. Таким образом, булев ранг матрицы $A$ не может превосходить значения $\min \{m, n\}$. Матрица $A$ называется матрицей полного булева ранга (или просто матрицей полного ранга), если ее булев ранг равен $\min \{m, n\}$. Понятие матриц полного ранга связано также с изучением простых элементов полугруппы булевых квадратных матриц порядка $n$ (см. [10]).

Настоящая работа посвящена изучению ограничений на число нулевых элементов $(0,1)$-матриц в зависимости от их булева ранга. Похожие исследования проводились и ранее; см., например, работы [7], [11], [12]. В работе [11] изучались возможные значения ранга булевой матрицы с фиксированным числом ненулевых элементов в ее строках и столбцах и были получены некоторые оценки на минимальное такое значение. В работе [12] были продолжены исследования множества значений ранга булевых матриц, начатые в [11], и было показано, что булев ранг матрицы размера $n \times n$, содержащей $k$ ненулевых элементов в каждой строке и каждом столбце, не может быть меньше, чем $n / k$. Точные значения минимума булева ранга были получены в работе [7] для квадратных матриц, содержащих $k$ ненулевых элементов в каждой строке и каждом столбце для $k \leqslant 4$ при любом натуральном $n$.

В настоящей работе мы также исследуем минимально возможные значения булева ранга при заданных условиях на число ненулевых элементов, но, в отличие от упомянутых работ, ограничиваем только число нулевых элементов 
в столбцах данной булевой матрицы. Исследуется вопрос о том, может ли матрица $A \in\{0,1\}^{n \times m}$ иметь полный булев ранг (равный $n$ ), если каждый из ее столбцов содержит не более чем $k$ нулевых элементов.

Вопрос. Рассмотрим множество тех матриц $A \in\{0,1\}^{n \times m}$, булев ранг которых равен $n$. При каких значениях переменной $k$ некоторый столбец любой матрицы $A$ обязательно содержит по крайней мере $k$ нулевых элементов?

Смягчим требование о полноте булева ранга в этом вопросе, заменив его рассмотрением матриц, имеющих сколь угодно большой булев ранг. Как показывает следующая теорема (см. также [13]), в этом случае мы можем гарантировать присутствие лишь одного нулевого элемента в столбцах матриц.

TеOPEма 1 (см. [14]). Пусть матрица $A \in\{0,1\}^{n \times m}$ задана соотношениями $A(i \mid j)=1$ при $i \neq j$ и $A(i \mid j)=0$ при $i=j$. Тогда булев ранг матриць $A$ равен наименвшему иелому числу $k$, для которого $n \leqslant\left(\begin{array}{c}{[k / 2]} \\ k\end{array}\right)$.

Требование полноты булева ранга оказывается значительно более сильным; в частности, из результатов настоящей работы будет следовать, что значение $k$ не может оставаться ограниченным при $n \rightarrow \infty$. Тем не менее будет показано, что отношение $k$ к $n$ становится сколь угодно малым при больших $n$. В работе будет найдено значение $k$, которое является асимптотически оптимальным при $n \rightarrow \infty$.

Исследование, результаты которого изложены в настоящей работе, было мотивировано изучением связи понятия булева ранга с понятием числа изоляции графа или $(0,1)$-матрицы (см. [7]). Введем понятие числа изоляции булевой матрицы, следуя работе [6].

ОПРЕДЕЛЕНИЕ 2. Числом изоляиии булевой матрицы $A$ называется наибольшее целое число $k$, для которого найдутся наборы индексов $r_{1}, \ldots, r_{k}$ и $c_{1}, \ldots, c_{k}$, для которых $A\left(r_{i} \mid c_{i}\right)=1$ при каждом $i$ и, кроме того, один из элементов $A\left(r_{i} \mid c_{j}\right)$ или $A\left(r_{j} \mid c_{i}\right)$ равен нулю при $i \neq j$.

Исследования взаимосвязи понятий булева ранга и числа изоляции начались довольно давно: в работе [7] было показано, что число изоляции заданной матрицы не превосходит ее булева ранга. В недавней статье [6] Бисли продолжил изучение поведения этих матричных инвариантов и показал, что число изоляции совпадает с булевым рангом, если какая-либо из этих величин не превосходит 2. Были также приведены примеры матриц, показывающие, что булев ранг может принимать сколь угодно большие значения, если даже изоляционное число фиксировано и равно 3. В настоящей работе показано, что аналогичное утверждение неверно для матриц полного булева ранга, иными словами, булевы матрицы полного ранга не могут иметь сколь угодно большого размера, если их числа изоляции ограничены.

Работа имеет следующую структуру. В $\S 2$ доказывается нижняя оценка на максимальное число нулевых элементов, содержащихся в столбцах булевых матриц полного ранга, и доказывается асимптотическая оптимальность этой 
оценки. А именно, показано, что оптимальное значение переменной $k$ в условиях поставленного выше вопроса принадлежит промежутку $[\sqrt{n} / 2-1, \sqrt{2 n}]$. В $\S 3$ обсуждаются приложения основных результатов; в частности, мы показываем (продолжая исследование, начатое Бисли в [6]), что булевы матрицы полного ранга не могут иметь сколь угодно большого размера при условии ограниченности чисел изоляции этих матриц.

\section{§ 2. Доказательство основного результата}

В этом параграфе мы даем ответ на поставленный вопрос и показываем, что значение $k=\sqrt{n} / 2-1$ удовлетворяет его условиям. В $\S 3$ будет доказана асимптотическая оптимальность этого значения. Для доказательства результатов параграфа нам потребуется следующее определение.

ОПРЕДЕЛЕНИЕ 3. БУлевой линейной оболочкой векторов $a_{1}, \ldots, a_{m} \in\{0,1\}^{n}$ называется множество всех булевых векторов вида

$$
\left(\lambda_{1} \otimes a_{1}\right) \oplus \cdots \otimes\left(\lambda_{m} \otimes a_{m}\right),
$$

где $\lambda_{1}, \ldots, \lambda_{m}$ выбираются из множества $\{0,1\}$.

Понятие булевой линейной оболочки дает важную характеризацию понятия булева ранга.

Лемма. Булев ранг матриць $A \in\{0,1\}^{n \times m}$ равен наименъшей возможной мощности такого множества $\alpha \subset\{0,1\}^{n}$, что все столби, матрици $A$ принадлежат булевой линейной оболочке векторов из множества $\alpha$.

ДокАЗАТЕЛЬство. Если булев ранг матрицы $A$ равен $k$, то в качестве $\alpha$ можно взять множество столбцов матрицы $B$ из определения 1 .

Предположим теперь, что столбцы матрицы $A$ принадлежат булевой линейной оболочке векторов $b_{1}, \ldots, b_{k}$. Тогда в силу определения 3 для любого $t \in\{1, \ldots, m\}$ найдутся такие $c_{1 t}, \ldots, c_{k t} \in\{0,1\}$, для которых линейная комбинация $\left(c_{1 t} \otimes b_{1}\right) \oplus \cdots \otimes\left(c_{k t} \otimes b_{k}\right)$ равна столбцу матрицы $A$ с номером $t$. Тогда, обозначив через $B$ матрицу, образованную столбцами $b_{1}, \ldots, b_{k}$, и задав матрицу $C \in\{0,1\}^{k \times m}$ соотношением $C(t \mid j)=c_{t j}$, получим $A=B \otimes C$.

Как следствие леммы получается следующее утверждение.

ПреДлОЖЕНИЕ 1. Если матрица $A^{\prime}$ получена из булевой матрицъ $А$ удалением одной строки или одного столбца, то булев ранг матрици $A^{\prime}$ равен или на единицу менъше, чем булев ранг матрищь $A$.

ДокАЗАТЕльство. Пусть матрица $A^{\prime}$ получена удалением столбца $a_{0}$ и ее столбцы принадлежат булевой линейной оболочке векторов $v_{1}, \ldots, v_{k}$. Тогда столбцы матрицы $A$ принадлежат булевой линейной оболочке векторов $a_{0}$, $v_{1}, \ldots, v_{k}$, поэтому результат следует из леммы.

Случай, когда матрица $A^{\prime}$ получена удалением строки, сводится к рассмотренному: равенство $C^{\top} \otimes B^{\top}=(B \otimes C)^{\top}$ показывает, что булевы ранги матриц инвариантны относительно транспонирования. 
Докажем теперь теорему, которая является важным шагом на пути к доказательству основного результата.

Теорема 2. Пустъ иелое число $q>1$ является степенъю простого числа. Если каждый столбеи, матрииы $A \in\{0,1\}^{q^{2} \times m}$ содержит не более чем $q-2$ нулевых элементов, то булев ранг матрицы $A$ не превосходит $q(q-1)$.

ДокАзАтельство. 1. Условимся обозначать индексы строк матрицы $A$ не с помощью первых $q^{2}$ натуральных чисел, а с помощью пар элементов поля $\mathbb{F}_{q}$; иными словами, строкам матрицы $A$ мы присвоим индексы из множества $\mathbb{F}_{q} \times \mathbb{F}_{q}$. Столбцы матрицы $A$ будут, как обычно, иметь индексы из множества $\{1, \ldots, m\}$. Введем в рассмотрение матрицу $B \in\{0,1\}^{q^{2} \times q(q-1)}$, строкам которой, как и строкам матрицы $A$, присвоим индексы из множества $\mathbb{F}_{q} \times \mathbb{F}_{q}$. Столбцам же матрицы $B$ присвоим индексы из множества $\mathbb{F}_{q} \times \mathbb{F}_{q}^{*}$, где $\mathbb{F}_{q}^{*}$ обозначает множество обратимых элементов поля $\mathbb{F}_{q}$; ясно, что $\left|\mathbb{F}_{q}^{*}\right|=q-1$. Зададим элементы матрицы $B$ как $B(k, l \mid g, h)=1$, если $l=g+k h$, и положим $B(k, l \mid g, h)=0$ в противном случае.

2. Теперь фиксируем произвольный элемент $v \in\{1, \ldots, m\}$ и рассмотрим некоторые элементы $k, l \in \mathbb{F}_{q}$, удовлетворяющие соотношению $A(k, l \mid v)=1$. В силу определения матрицы $B$ при каждом $s \in \mathbb{F}_{q}^{*}$ выполнено условие $B(k, l \mid-s k+l, s)=1$. Из определения матрицы $B$ также следует, что если элементы $k^{\prime}, l^{\prime} \in \mathbb{F}_{q}$ таковы, что $\left(k^{\prime}, l^{\prime}\right) \neq(k, l)$, то не более чем один элемент $s^{\prime} \in \mathbb{F}_{q}^{*}$ удовлетворяет условию $B\left(k^{\prime}, l^{\prime} \mid-s^{\prime} k+l, s^{\prime}\right)=1$. Согласно условию теоремы не более чем $q-2$ пар $\left(k^{\prime}, l^{\prime}\right)$ удовлетворяют условию $A\left(k^{\prime}, l^{\prime} \mid v\right)=0$. В силу принципа Дирихле не более чем $q-2$ элементов $s^{\prime} \in \mathbb{F}_{q}^{*}$ удовлетворяют условиям $B\left(k^{\prime}, l^{\prime} \mid-s^{\prime} k+l, s^{\prime}\right)=1$ и $A\left(k^{\prime}, l^{\prime} \mid v\right)=0$ при каких-либо $k^{\prime}$ и $l^{\prime}$. Таким образом, найдется такой элемент $\widehat{s}=\widehat{s}(k, l, v) \in \mathbb{F}_{q}^{*}$, что $B\left(k^{\prime}, l^{\prime} \mid-\widehat{s} k+l, \widehat{s}\right)=0$ при любых $v, k^{\prime}$ и $l^{\prime}$, удовлетворяющих условию $A\left(k^{\prime}, l^{\prime} \mid v\right)=0$.

3. Теперь рассмотрим множество всех пар $(k, l)$, для которых $A(k, l \mid v)=1$, и обозначим через $\mathscr{S}_{v}$ множество всех пар $(-\widehat{s}(k, l, v) k+l, \widehat{s}(k, l, v))$. Пункт 2 показывает, что $v$-й столбец матрицы $A$ равен булевой сумме столбцов матрицы $B$ с индексами из множества $\mathscr{S}_{v}$. Иными словами, каждый столбец матрицы $A$ представим как булева линейная комбинация столбцов матрицы $B$, поэтому доказываемый результат следует теперь из леммы.

Теперь мы можем доказать неравенство, связывающее размер матрицы с количеством нулевых элементов в ее столбцах.

Tеорема 3. Пусть $A \in\{0,1\}^{n \times m}$ - матрица булева ранга $n$. Тогда некоторый столбеи, матрицы $А$ содержит по крайней мере $q-1$ нулевых элементов, где q - наибольшее челое число, которое является степенью простого и удовлетворяет условию $q^{2}-q+1 \leqslant n$.

ДокАЗАТЕЛЬСтво. Предположим противное. Если $n \geqslant q^{2}$, то обозначим через $A^{\prime}$ матрицу, полученную из $A$ удалением произвольных $n-q^{2}$ строк. В силу теоремы 2 булев ранг матрицы $A^{\prime}$ не превосходит $q^{2}-q$, поэтому в силу предложения матрица имеет булев ранг, не больший $n-q<n$. Противоречие. 
Если же $n<q^{2}$, то рассмотрим матрицу $A^{\prime \prime}$, полученную из $A$ добавлением $q^{2}-n$ строк, состоящих из элементов 1 . В силу теоремы 2 матрица $A^{\prime \prime}$ имеет булев ранг, не превосходящий $q^{2}-q$. Матрица $A$ является подматрицей матрицы $A^{\prime \prime}$, и поэтому ее булев ранг тоже не превосходит $q^{2}-q<n$. Полученное противоречие завершает доказательство.

Чтобы получить оценку числа нулевых элементов в явном виде, нам потребуется следующий классический результат теории чисел.

ТЕОрема 4 (см. [15]). Для любого иелого положительного числа $k$ найдется простое число $p$, удовлетворяющее условию $k<p \leqslant 2 k$.

Теперь мы можем сформулировать основной результат статьи.

Теорема 5. Пусть $A \in\{0,1\}^{n \times m}-$ матрица булева ранга $n$. Тогда некоторый столбеи, матрицы $A$ содержит по крайней мере $\sqrt{n} / 2-1$ нулевых элементов.

Поскольку в силу теоремы 4 существует простое число $p$, большее $[\sqrt{n} / 2]$ и не превосходящее $[\sqrt{n}]$, то достаточно применить теорему 3 .

\section{§ 3. Оптимальность основного результата и некоторые его приложения}

Этот параграф посвящен обсуждению некоторых следствий и приложений теоремы 5 , основного результата работы. Сначала покажем, что результат теоремы 5 асимптотически оптимален при $n \rightarrow \infty$.

ПреДЛОЖЕНИЕ 2. Пусть $t$ - положительное целое число, положим также $f(t)=\left(t^{2}+3 t+4\right) / 2$. Обозначим через $\Psi$ множество всех $t$-элементных подмножеств множества $\psi=\{1, \ldots, f(t)\}$. Определим булеву матрииу $A=A(t)$ следующим образом: присваивая ее строкам индексы из множества $\psi$, столбцам - из множества $\Psi$, положим $A(i \mid I)=0$, если $i \in I, u$ $A(i \mid I)=1$ в противном случае. Тогда булев ранг матрицы $A$ равен $f(t)$.

ДоказАтельство. Для доказательства требуется проверить, что матрицы $B \in\{0,1\}^{f(t) \times(f(t)-1)}$ и $C \in\{0,1\}^{(f(t)-1) \times|\Psi|}$ не могут удовлетворять условию $A=B \otimes C$. Случай $t=1$ соответствует примеру 2.4 работы [6], а также может быть разобран непосредственно с помощью определения 1.

В случае $t>1$ продолжим доказательство по индукции. Предположим, что матрицы $B$ и $C$ удовлетворяют условию $B \otimes C=A$. В соответствии с определением матрицы $A$ присвоим строкам матрицы $B$ индексы из множества $\psi$, столбцам матрицы $C$ - индексы из множества $\Psi$. Столбцам матрицы $B$ так же, как и строкам матрицы $C$, присвоим индексы из множества $\{1, \ldots, f(t)-1\}$. Рассмотрим два возможных случая.

1. Предположим, что любая строка матрицы $B$ содержит не более чем $t$ элементов 1. Поскольку число строк матрицы $B$ больше чем число ее столбцов, найдется такой индекс $u \in \psi$, что для любого индекса $v$, удовлетворяющего 
условию $B(u \mid v)=1$, условие $B\left(u^{\prime}(v) \mid v\right)=1$ также выполняется при некотором $u^{\prime}(v) \neq u$. Множество всех индексов $v$, удовлетворяющих условию $B(u \mid v)=1$, обозначим через $V$. Определение умножения булевых матриц показывает, что если $A(u \mid \nu)=1$ при некотором $\nu \in \Psi$, то $A\left(u^{\prime}(v) \mid \nu\right)=1$ для некоторого $v \in V$. С другой стороны, в силу нашего предположения мощность множества $V$ не превосходит $t$, поэтому найдется множество $\mathscr{I} \in \Psi$, удовлетворяющее условиям $u \notin \mathscr{I}$ и $u^{\prime}(v) \in \mathscr{I}$ при любом $v \in V$. В этом случае $A(u \mid \mathscr{I})=1$ и $A\left(u^{\prime}(v) \mid \mathscr{I}\right)=0$ при любом $v \in V$. Противоречие.

2. Теперь предположим, что некоторая строка (обозначим ее номер через $\mu$ ) матрицы $B$ содержит по крайней мере $t+1$ элементов, равных 1. Опять обозначим через $V$ множество всех индексов $v$, удовлетворяющих условию $B(\mu \mid v)=1$; через $\bar{V}$ обозначим множество всех индексов $\bar{v}$, удовлетворяющих условию $B(\mu \mid \bar{v})=0$. Через $\psi^{\prime}$ обозначим произвольное подмножество множества $\psi \backslash\{\mu\}$, состоящее из $f(t-1)$ элементов; обозначим через $\Psi^{\prime}$ множество всех $(t-1)$-элементных подмножеств множества $\psi^{\prime}$. Любое множество $\mathscr{J} \in \Psi^{\prime}$ теперь удовлетворяет условию

$$
\bigoplus_{k \in V \cup \bar{V}} B(\mu \mid k) \otimes C(k \mid \mathscr{J} \cup\{\mu\})=\mathrm{A}(\mu \mid \mathscr{J} \cup\{\mu\})=0,
$$

поэтому $C(v \mid \mathscr{J} \cup\{\mu\})=0$ для любого $v \in V$.

Таким образом, для любых $\omega \in \psi^{\prime}$ и $\mathscr{J} \in \Psi^{\prime}$ выполняется условие

$$
\bigoplus_{k \in \bar{V}} B(\omega \mid k) \otimes C(k \mid \mathscr{J} \cup\{\mu\})=\mathrm{A}(\omega \mid \mathscr{J} \cup\{\mu\}) .
$$

Заметим, что подматрица, образованная строками матрицы $A$ с индексами из множества $\psi^{\prime}$ и столбцами с индексами из множества $\mathscr{J} \cup\{\mu\}$, совпадает с матрицей $A(t-1)$ с точностью до переобозначения индексов столбцов и строк. Последнее равенство поэтому показывает, что булев ранг матрицы $A(t-1)$ не превосходит мощности множества $\bar{V}$, т.е. не превосходит $f(t)-t-2$. Пользуясь предположением индукции, получаем неравенство $f(t-1) \leqslant f(t)-t-2$ и приходим к противоречию.

Таким образом, каждая из возможностей 1 и 2 была сведена нами к противоречию. Значит, матрицы $B \in\{0,1\}^{f(t) \times(f(t)-1)}$ и $C \in\{0,1\}^{(f(t)-1) \times|\Psi|}$ на самом деле не могут удовлетворять условию $A=B \otimes C$.

Докажем теперь асимптотическую оптимальность результата теоремы 5.

СледствиЕ. Каково бы ни было челое положительное число $n$, найдется $(0,1)$-матрица булева ранга $n$ размера $n \times m$, любой столбеи, которой содержит не более, чем $\sqrt{2 n}$ нулевых элементов.

Для доказательства используем предложение 2 для $t=[\sqrt{2 n}]$ и затем применим предложение 1.

Теорема 5 и следствие показывают, что оптимальное значение величины $k$ из вопроса в $\S 1$ принадлежит промежутку $[\sqrt{n} / 2-1, \sqrt{2 n}]$. Иными словами, оптимальное $k$ имеет порядок роста $\Theta(\sqrt{n})$ при $n \rightarrow \infty$. 
Теперь мы покажем, как теорема 5 может быть применена к исследованию поведения различных инвариантов булевых матриц. Об одном из них - числе изоляции булевой матрицы - было подробно рассказано во введении; минорный и тропический ранги, функции, обобщающие классическое понятие ранга на булев случай, представляют собой другие примеры таких инвариантов. Эти функции определяются с помощью понятия вырожденности булевых матриц.

ОПРЕДЕЛЕНИЕ 4 (см. [2]). Булева матрица $U \in\{0,1\}^{k \times k}$ называется d-невърожденной, если равенство

$$
U(1 \mid \sigma(1)) \otimes \cdots \otimes U(k \mid \sigma(k))=1
$$

выполняется для некоторой перестановки $\sigma$ из симметрической группы $\mathscr{S}_{k}$ и не выполняется ни для какой перестановки, четность которой отлична от четности $\sigma$.

Матрица $U$ называется тропически невырожденной, если равенство

$$
U(1 \mid \varphi(1)) \otimes \cdots \otimes U(k \mid \varphi(k))=1
$$

выполняется для единственной перестановки $\varphi \in \mathscr{S}_{k}$.

Минорным рангом (см. [16]) (соответственно тропическим рангом; см. [2]) булевой матрицы $A$ называется наибольший размер $\mathrm{d}$-невырожденной (соответственно тропически невырожденной) квадратной подматрицы матрицы $A$. Хотя булев ранг матрицы может быть сколь угодно велик при ограниченных значениях перечисленных инвариантов (см. [2], [6]), оказывается, что аналогичное утверждение перестает быть верным в предположении полноты булева ранга. Оказывается верной следующая теорема.

Теорема 6. Пусть $k, n$ и $m$ - целые положительные числа, $n \geqslant 16^{2^{k}-1}$. Если матрица $A \in\{0,1\}^{n \times m}$ имеет булев ранг $n$, то найдутся семейства индексов $r_{1}, \ldots, r_{k} u c_{1}, \ldots, c_{k}$, для которых $A\left(r_{i} \mid c_{i}\right)=1 u A\left(r_{j} \mid c_{i}\right)=0$ nри $j>i$.

ДокАЗАтЕльство. При $k=1$ утверждение тривиально; предположим, что $k>1$, и продолжим доказательство индукцией по $k$. В этом случае из теоремы 5 следует, что некоторый столбец (обозначим его номер через $c_{1}$ ) матрицы $A$ содержит по крайней мере $\sqrt{n} / 4 \geqslant 16^{2^{k-1}-1}$ нулевых элементов. Ясно, что удаление нулевых столбцов не влияет на ранг матрицы; поэтому мы можем считать, что $A\left(r_{1} \mid c_{1}\right)=1$ при некотором $r_{1} \in\{1, \ldots, n\}$. Обозначим через $A^{\prime}$ матрицу, полученную из $A$ удалением столбцов с индексами $\rho$, удовлетворяющими условию $A\left(\rho \mid c_{1}\right)=1$. По предположению индукции для матрицы $A^{\prime}$ найдутся такие семейства индексов $r_{2}, \ldots, r_{k}$ и $c_{2}, \ldots, c_{k}$, что $A\left(r_{i} \mid c_{i}\right)=1$ и $A\left(r_{j} \mid c_{i}\right)=0$ при всех $i$ и $j$, удовлетворяющих условию $j>i>1$. Поскольку индексы $r_{2}, \ldots, r_{k}$ являются номерами строк матрицы $A^{\prime}$, имеем также $A\left(r_{g} \mid c_{1}\right)=1$ для каждого $g>1$.

Теперь мы можем доказать следующую теорему, которая дает верхнюю оценку размера матрицы полного булева ранга в терминах других рассмотренных нами инвариантов булевой матрицы. 
Теорема 7. Пусть матрица $A \in\{0,1\}^{n \times m}$ имеет полный булев ранг. Если минорный или тропический ранги либо число изоляиии матрицы $A$ менвше $k$, mo

$$
\min \{n, m\}<16^{2^{k}-1} .
$$

ДоКАЗАТЕЛЬСтво. Предположим противное и рассмотрим подматрицу $A^{\prime}$ матрицы $A$ из теоремы 6 , образованную строками с индексами $r_{1}, \ldots, r_{k}$ и столбцами с индексами $c_{1}, \ldots, c_{k}$. В силу определения 4 матрица $A^{\prime}$ d-невырождена и тропически невырождена, поэтому минорный и тропический ранги матрицы $A$ не могут быть меньше чем $k$. Кроме того, в силу определения 2 число изоляции матрицы $A$ не может быть меньше чем $k$. Далее, булев, минорный, тропический ранги и число изоляции инвариантны относительно транспонирования матриц, поэтому результат следует из теоремы 6.

\section{Список литературы}

[1] L. B. Beasley, N. J. Pullman, "Semiring fank versus column rank", Linear Algebra Appl., 101 (1988), 33-48.

[2] M. Akian, S. Gaubert, A. Guterman, "Linear independence over tropical semirings and beyond", Tropical and idempotent mathematics, Contemp. Math., 495, Amer. Math. Soc., Providence, RI, 2009, 1-38.

[3] J. E. Cohen, U. G. Rothblum, "Nonnegative ranks, decompositions, and factorizations of nonnegative matrices", Linear Algebra Appl., 190 (1993), 149-168.

[4] M. Develin, F. Santos, B. Sturmfels, "On the rank of a tropical matrix", Combinatorial and computational geometry, Math. Sci. Res. Inst. Publ., 52, Cambridge Univ. Press, Cambridge, 2005, 213-242.

[5] P. C. Fishburn, P. L. Hammer, "Bipartite dimensions and bipartite degrees of graphs", Discrete Math., 160:1-3 (1996), 127-148.

[6] L. B. Beasley, "Isolation number versus Boolean rank", Linear Algebra Appl., 436:9 (2012), 3469-3474.

[7] D. A. Gregory, N. J. Pullman, K. F. Jones, J. R. Lundgren, "Biclique coverings of regular bigraphs and minimum semiring ranks of regular matrices", J. Combin. Theory Ser. B, 51:1 (1991), 73-89.

[8] G. Shu, D. Lee, M. Yannakakis, "A note on broadcast encryption key management with applications to large scale emergency alert systems", Proceedings of the 20th International Parallel and Distributed Processing Symposium, IEEE Computer Society, Washington, 2006.

[9] A. Ene, W. G. Horne, N. Milosavljevic, P. Rao, R. Schreiber, R. E. Tarjan, "Fast exact and heuristic methods for role minimization problems", Proceedings of the 13th ACM Symposium on Access Control Models and Technologies, ACM, New York, 2008.

[10] D. de Caen, D. A. Gregory, "Primes in the semigroup of Boolean matrices", Linear Algebra Appl., 37 (1981), 119-134.

[11] R. A. Brualdi, R. Manber, J. A. Ross, "On the minimum rank of regular classes of matrices of zeros and ones", J. Combin. Theory Ser. A, 41:1 (1986), 32-49.

[12] K. A. S. Hefner, J. R. Lundgren, "Minimum matrix rank of $k$-regular $(0,1)$ matrices", Linear Algebra Appl., 133 (1990), 43-52.

[13] S. Bezrukov, D. Froncek, S. J. Rosenberg, P. Kovar, "On biclique coverings", Discrete Math., 308:2-3 (2008), 319-323. 
[14] D. de Caen, D. A. Gregory, N. J. Pullman, "The Boolean rank of zero-one matrices", Proceedings of the Third Caribbean Conference on Combinatorics and Computing (Bridgetown, 1981), Univ. West Indies, Cave Hill Campus, Barbado, 1981, 169-173.

[15] M. Aigner, G. M. Ziegler, Proofs from the book, Springer-Verlag, Berlin, 1999.

[16] В.Б. Поплавский, "О рангах, классах Грина и теории определителей булевых матриц", Дискрет. матем., 20:4 (2008), 42-60; англ. пер.: V. B. Poplavskii, "On ranks, Green classes, and the theory of determinants of Boolean matrices", Discrete Math. Appl., 18:6 (2008), 641-658.

\section{Ярослав Николаевич Шитов} (Yaroslav N. Shitov)

Национальный исследовательский университет 14.07.2012 и 13.03 .2013

"Высшая школа экономики"

E-mail: yaroslav-shitov@yandex.ru 